\title{
Improving Stroke Care in Bhutan
}

\author{
Yangchen Yangchen ${ }^{\mathrm{a}}$ Tashi Tenzin $^{\mathrm{b}}$ Nar Bahadur Raic ${ }^{\mathrm{c}}$ Sonam Yangzom ${ }^{\mathrm{d}}$ \\ Narayanaswamy Venketasubramanian ${ }^{\mathrm{e}}$ \\ aDepartment of Internal Medicine, Jigme Dorji Wangchuck National Referral, Hospital, Thimphu, Bhutan; \\ ${ }^{\mathrm{b}}$ Department of Surgery, Jigme Dorji Wangchuck National Referral Hospital, Thimphu, Bhutan; 'Department of \\ Emergency Medicine, Central Regional Referral Hospital, Mongar, Bhutan; dDepartment of Medicine, Eastern

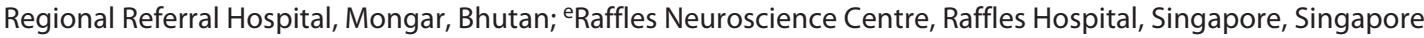

\section{Keywords}

Stroke care initiative $\cdot$ Stroke care quality $\cdot$ BRAINS

implementation research $\cdot$ Bhutan

\begin{abstract}
Developing nations face the double burden of communicable and noncommunicable diseases. Bhutan is a developing country and has achieved significant milestones in its health indicators. Increasing burden of stroke and the lack of evidence-based stroke care system in the country's hospitals are proving to be a challenge to provide quality stroke care. Despite the logistical challenge of referring stroke patients on time, lack of trained health care professionals and resources, Bhutan has recently started various initiatives to improve stroke care with the help of WHO-SEARO, WHO-Geneva, and Christian Medical College, Ludhiana, India.

(c) 2022 The Author(s)

Published by S. Karger AG, Basel
\end{abstract}

\section{Introduction}

Bhutan is a developing nation in the eastern Himalayas with a population of 741,672 in 2019. The capital is Thimphu City (Fig. 1). Elevation rises from $200 \mathrm{~m}$ in the southern foothills to more than $7,000 \mathrm{~m}$, which poses unique challenges in health services delivery. Health care is pro-

Karger@karger.com www.karger.com/cee

Karger"
(C) 2022 The Author(s)

Published by S. Karger AG, Basel

This is an Open Access article licensed under the Creative Common Attribution-NonCommercial-4.0 International License (CC BY-NC) (http://www.karger.com/Services/OpenAccessLicense), applicable to the online version of the article only. Usage and distribution for commercial purposes requires written permission. vided free by the Royal Government of Bhutan, including referrals to Indian Hospitals. Bhutan has made significant progress in improving the health and quality of life of its population in recent decades, achieving almost all of its millennium development goals [1].

Over the span of 5 decades the life expectancy has more than doubled to 70.2 years in 2017, from 32.4 years in 1960. While Bhutan still has a young population with median age of 26.9 years, the declining TFR and mortality rates are growing concerns as they result in population aging and rising dependency which call for strengthening of the existing health system for chronic care [1].

\section{Stroke Epidemiology}

Stroke has emerged as a paramount health concern worldwide and is now the second leading cause of death and third leading cause of disability [2]. In recent years, the disease pattern in Bhutan has been changing with an increasing incidence of noncommunicable diseases, like cancer and stroke. According to the Annual Health Bulletin of Bhutan published by the Ministry of Health in 2019, stroke was the 5th highest cause of death in the country [3]. According to a recent study, mean lifetime risk of stroke in 2016 was $16.8 \%$ (confidence interval, [CI]: 15.2-18.4) for all strokes, 5.4\% (CI: 4.7-6.1) for hemorrhagic stroke, and $12.0 \%$ (CI: 10.6-13.5) for ischemic stroke. The mean percentage changes in lifetime risk 


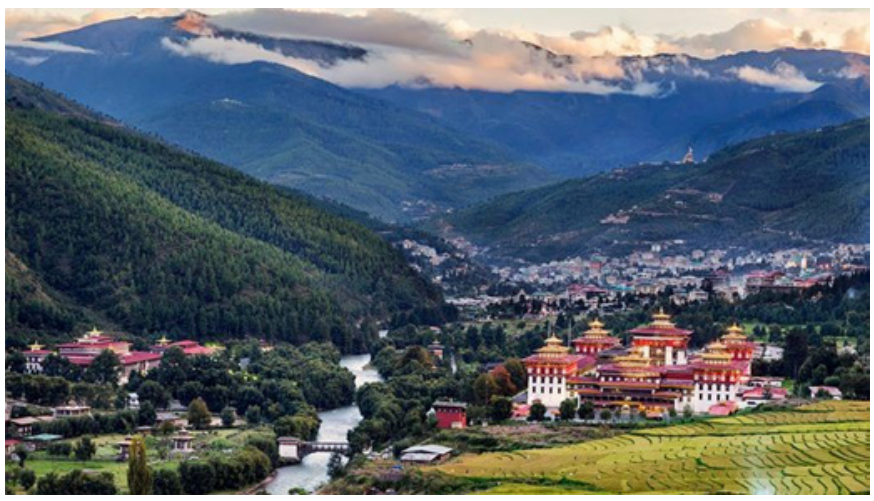

Fig. 1. Thimphu city, capital of Bhutan. (c) Andrew Stranovsky Photography - Moment/Getty Image.

of stroke from 1990 to 2016 for total, hemorrhagic and ischemic strokes were $1.5 \%,-0.4 \%$, and $2.1 \%$, respectively [4]. Age- and sex-standardized mortality and disability adjusted life years due to stroke are lower than most other countries in South Asia [5].

\section{Stroke Care Systems}

The country has a three-tiered government health system, which includes the Jigme Dorji Wangchuck National Referral Hospital (JDWNRH) at the tertiary level (Fig. 2), two regional referral hospitals, the Eastern Regional Referral Hospital, and the Central Regional Hospital covering the eastern and southern parts of the country, respectively, district hospitals at the secondary level and Basic Health Units and out-reach clinics at the primary level. For advanced care like renal transplant, etc., patients are referred to India through a referral process and the costs borne by the government [1].

Hospitals in Bhutan do not have stroke system of care in place. At present, there are no neurologists in the hospitals in the whole of the country. There is a general lack of awareness of the need to establish stroke units and treat stroke patients with evidence-based protocols among health care workers. Stroke patients are admitted in the Internal Medicine department due to lack of stroke units. There is lack of protocol-based management of stroke patients in these wards. In the emergency department, there is no dedicated acute stroke bed or an acute stroke management protocol in place. As a result, hardly any eligible ischemic stroke patients get thrombolytic therapy and not a single stroke patient is managed in stroke units with evidence-based treatments including FeSS protocol, DVT prophylaxis, antithrombotic/anticoagulation, rehabilitation and follow-up after discharge, etc.

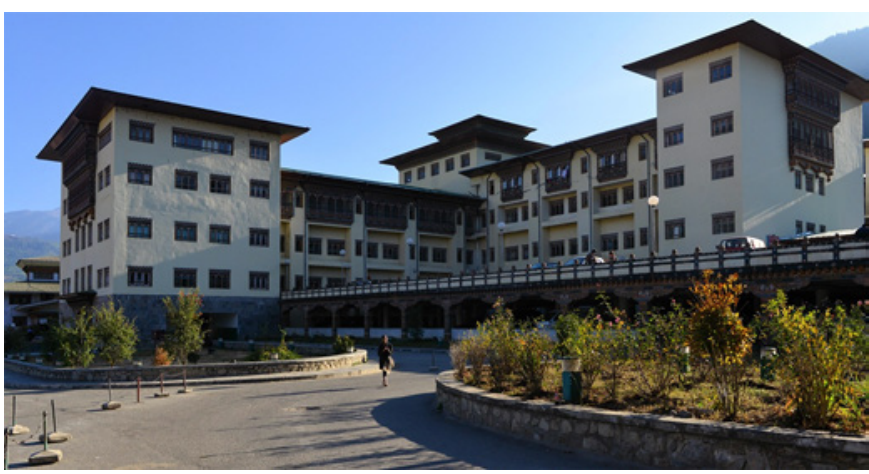

Fig. 2. Jigme Dorji Wangchuck National Referral Hospital, Thimphu, Bhutan. (CWikimedia Commons.

\section{Enhancing Stroke Care}

Cognizant of the rising burden of stroke in the country and the present level of stroke care in the country's hospitals, a team of health workers from JDWNRH, Eastern Regional Referral Hospital, and Central Regional Referral Hospital initiated various activities to improve stroke care. Extensive collaboration and training of health workers have been done with the help of the Department of Neurology and Physiotherapy, Christian Medical College, Ludhiana, Punjab, India and WHO-SEARO, and Geneva over the last one and half years, despite the challenges of the Covid-19 pandemic. An Implementation Research protocol on stroke care improvement, titled BRAINS (Bhutan's Response Against Increasing Number of Stroke) has been developed and is in the final stages of implementation. With this implementation research, we plan to establish stroke units, facilitate adoption of acute stroke management protocols in the three referral hospitals in the country, raise awareness of stroke, importance of evidence-based stroke care among the health workers and policy makers and eventually the general public. With these initiatives, we hope to increase the quality of stroke care in Bhutan. We hope to be able to publish the research soon and identify areas for further improvement.

We are also happy to note that stroke is gradually receiving attention among the public and policy makers with Civil Society Organizations (CSOs) like Bhutan Stroke Foundation getting established in 2019 and registered as a CSO recently. Our medical team provides the technical support to the CSO and helps them in raising awareness of stroke to the public, including during the World Stroke Day observations annually.
Yangchen/Tenzin/Rai/Yangzom/ Venketasubramanian 


\section{Conclusions}

Despite the lack of trained stroke specialists and neurologists in the country, we hope to establish evidencebased acute stroke care in the three referral hospitals of the country with the implementation of the BRAINS implementation research. We aim to utilize the existing robust patient referral system already in place with GPS equipped ambulances, and helicopter medical evacuations for acute stroke patients in order to offer thrombolytic therapy to eligible ischemic stroke patients. Our future aspirations include getting the three referral hospitals certified as Primary Stroke Centers and eventually establish a comprehensive stroke center at the JDWNRH in the capital city, Thimphu.

\section{Acknowledgments}

We would like to thank Professor Pandian and his team at CMC, Ludhiana, Dr. Gampo at WHO-SEARO, Dr. Ruitai at WHO-Geneva for helping the Bhutanese health team to spearhead this extremely important initiative to improve the quality of stroke care in Bhutan.

\section{Statement of Ethics}

No statement of ethics is needed as this is not a research study on human subjects.

\section{Conflict of Interest Statement}

We declare that there is no conflict of interest.

\section{Funding Sources}

We declare that there are no funding disclosures to be made.

\section{Author Contributions}

Yangchen Yangchen, MD - wrote the paper and gave final approval. Tashi Tenzin, MS - provided critical review and gave final approval. Nar Bahadur. Rai, MD - provided critical review and gave final approval. Sonam Yangzom, MD - provided critical review and gave final approval. Narayanaswamy Venketasubramanian, $\mathrm{MD}$ - provided critical review, gave final approval, and prepared the paper for submission.

\section{Data Availability Statement}

All data mentioned in this paper are taken from sources in the public domain. Thus, no data availability statement is needed.

\section{References}

1 National Statistics Bureau. Population Projections Bhutan. 2017-2047, (2019).

2 Institute for Health Metrics and Evaluation. Global Health Data Exchange. GBD compare Viz hub. http://vizhub.healthdata.org/gbdcompare (accessed November 24, 2019.

3 Ministry of Health. National Health Accounts, Bhutan Fiscal Years 2014-15 \& 201516.
4 GBD 2016 Lifetime Risk of Stroke Collaborators, Feigin VL, Nguyen G, Cercy K, Johnson $\mathrm{CO}$, Alam T, et al. Global, regional, and country-specific lifetime risks of stroke, 1990 and 2016. N Engl J Med. 2018;379:2429-37.

5 Venketasubramanian N, Yoon BW, Pandian J, Navarro JC. Stroke epidemiology in South, East, and South-East Asia: a review. J Stroke. 2017;19:286-94. 